\title{
Default options: a powerful behavioral tool to increase COVID-19 contact tracing app acceptance in Latin America?
}

\author{
Cynthia Boruchowicz ${ }^{1 \star}$, Florencia Lopez Boo ${ }^{2}$, Benjamin Roseth ${ }^{2}$ and Luis Tejerina ${ }^{2}$ \\ ${ }^{1}$ School of Public Policy, University of Maryland, College Park, MD, USA and ${ }^{2}$ Inter-American \\ Development Bank, Washington, DC, USA \\ ${ }^{*}$ Corresponding to: E-mail: cynthiab@umd.edu
}

\begin{abstract}
Given the rates of transmission of COVID-19, relying only on manual contact tracing might be infeasible to control the epidemic without sustained costly lockdowns or rapid vaccination efforts. In the first study of its kind in Latin America, we find through a phone survey of a nationally representative sample of ten countries that an opt-out regime (automatic installation) increases self-reported intention to accept a contact tracing app with exposure notification by 22 percentage points compared to an opt-in regime (voluntary installation). This effect is triple the size and of opposite sign of the effect found in Europe and the United States, potentially due to lower concerns regarding privacy and lower levels of interpersonal trust. We see that an opt-out regime is more effective in increasing willingness to accept for those who do not trust the government or do not use their smartphones for financial transactions. The local severity of the pandemic does not affect our results, but feeling personally at risk increases intent to accept such apps in general. These results can shed light on the use of default options not only for contact tracing apps but in public health overall in the context of a pandemic in Latin America.
\end{abstract}

Keywords: contact tracing apps; Latin America; default options; trust; COVID-19

JEL Classification codes: D12; D91; I12; I18

\section{Introduction}

The first case of COVID-19 was confirmed in Latin America on February 26, 2020, in Brazil. By mid-March, most countries in the region had adopted restrictive measures like banning international flights, prohibiting public mass gatherings, suspending in-person education, and closing restaurants and bars - in addition to communicating preventive measures aimed at keeping social distance, washing hands and, later, face covering. On May 22, 2020, the World Health Organization (WHO) declared South America the new epicenter of the pandemic (Feuer, 2020). To cope with the pandemic, as well as manage the reopening of their economies, countries around the globe have relied on technological tools in mobile phones for contact tracing, (c) The Author(s), 2021. Published by Cambridge University Press 
such as Bluetooth Low Energy (BLE). Such technology can be used to detect and inform users when they have been in close-range physical contact with individuals who have tested positive for COVID-19 (and have informed their condition on the system). As such, it facilitates contact tracing between participating devices while allowing for the protection of private data (Bay et al., 2020; DP-3T Project, 2020; Troncoso et al., 2020). Testing, contact tracing, and quarantine/isolation were and continue to be key strategies to contain the spread of an infectious disease for which vaccination is not (widely) available. The use of contact tracing apps with exposure notifications ${ }^{1}$ could alleviate the necessity of in-person contact tracing if enough people use it (Ferretti et al., 2020). With rates of uptake as low as $10 \%$, contact tracing apps with exposure notifications are considered a preventive tool that can help save lives (Abueg et al., 2020; Ferretti et al., 2020; Servick, 2020) and control the epidemic without further sustained costly lockdowns (Abeler et al., 2020) or rapid vaccination efforts. Though contextual factors like virus caseloads and scope of manual contact tracing efforts can affect the minimum uptake rates to guarantee effectiveness of the apps, greater uptake improves effectiveness. In Supplementary Appendix Section A, we provide a detailed description on the technical aspects of contact tracing apps and how they compare to manual tracing.

Latin America and the Caribbean present a particularly challenging setting for the uptake of contact tracing apps with exposure notifications. Citizens have low levels of trust in government (important because apps are provided by public institutions), low trust in fellow citizens (important given that most apps require the infected person to change their own status within the application; so if citizens do not trust others to take that step, then they have less reason to download and use the app), and high degrees of concern regarding data privacy (Roseth et al., 2018). Furthermore, most countries have weak data protection frameworks, and low-income individuals might also be concerned about overloading their data plans (Roseth \& Porrúa, 2021). Therefore, acceptability and uptake of contact tracing apps with exposure notifications are key (Anglemyer et al., 2020). For this reason, the Inter-American Development Bank (IDB) conducted a phone survey of a nationally representative sample of ten countries with 1000 observations per country, to understand, among others, the perceptions from the population of Latin America regarding the use of these types of apps with a focus on issues like trust and data usage. In this article, we use this survey to measure the self-reported intention to accept a contact tracing app with exposure notification depending on whether it is opt-in or opt-out. We achieve this by taking advantage that randomly in each country, half of the respondents in the survey were assigned to a questionnaire that asked about a hypothetical contact tracing app with exposure notification that the individual could download if $\mathrm{s} /$ he wishes, and half were assigned to a questionnaire about a default app that would be installed automatically (and can be uninstalled). To our knowledge, this is the first

\footnotetext{
${ }^{1}$ The WHO and the Pan American Health Organization (PAHO) use the term exposure notification apps. The term contact tracing apps with exposure notification follows current literature on mobile phone apps that can trace the close contacts of infected individuals (see Abeler et al., 2020; Abueg et al., 2020; Akinbi et al., 2020; Altmann et al., 2020; Anglemyer et al., 2020; Ayres et al., 2020; Bradshaw et al., 2020; Frimpong et al., 2020; Trang et al., 2020).
} 
study to apply behavioral insights to the issue of intention to accept contact tracing apps with exposure notifications in Latin America, and the first to use survey experimental data to analyze the use of default options overall. We find that the opt-out option increases the self-reported intention to accept a contact tracing app with exposure notification by almost 22 percentage points compared with the opt-in one. This is opposite to what was found by Altmann et al. (2020) in developed countries through a non-experimental online survey targeted at intent to download/not remove contact tracing apps. In that case, the opt-in option seems to do a better job at increasing possible uptake. Our assumption is that this difference is potentially due to a higher value placed in privacy, and higher interpersonal trust in Europe compared with Latin America.

The severity and urgency of the COVID-19 pandemic meant that several studies relied on self-reported behaviors and perceptions to provide policy analysis and recommendations. ${ }^{2}$ Consequently, while the information collected through this survey refers to a self-reported intention to either download or not uninstall the application, and therefore, whether that translates into observable actions is beyond the scope of this article, it can shed light on the use of default options in the context of the pandemic in the region. The previous is particularly relevant considering that a randomized controlled trial of a contact tracing app with exposure notification is not feasible (Wang, 2021) and that different Latin American countries like Ecuador and Uruguay are already implementing this type of apps. In the UK, it has been estimated that for every $1 \%$ increase in app users, the number of COVID-19 infections can be reduced up to $2.3 \%$ (Wymant et al., 2021). Translating such results to Latin America, where the pandemic is far from over [150,000 new daily infections as of July 2021 (Reuters, 2021)], is key.

\section{Behavioral Sciences, health, and technology Use of default options on health interventions}

Default options can be described as '... events or conditions set into place when no alternatives are actively chosen' (Halpern, 2018, p. 2). As such, they are expected to produce changes in behavior because people tend to stick with the default option, while giving individuals freedom of choice by allowing them to opt-out (Choi et al., 2004; Sunstein \& Reisch, 2014). Setting default options has been used in a variety of settings such as car insurance plan choices (Johnson et al., 1993), car option purchases (Park et al., 2000), consent to receive e-mail marketing (Johnson et al., 2003), 401(K) plans (Bernheim et al., 2015), and a variety of environmental preservation efforts (Sunstein \& Reisch, 2014; Kaiser et al., 2020; Susntein, 2020). Regarding health-oriented decisions, default options have been shown to modify multiple behaviors - including increasing vaccination rates (Chapman et al., 2010), the choice of comfort-oriented end-of-life care (Halpern et al., 2013), consumption of fruits and vegetables (Just \& Price, 2013), and the willingness to be an organ donor (Johnson

\footnotetext{
${ }^{2}$ See, for example, Altmann et al. (2020), Bradshaw et al. (2020), and Ayres et al. (2020) for studies on contact tracing apps; or Wise et al. (2020), Glöckner et al. (2020), Everett et al. (2020), and Lunn et al. (2021) for those on preventive behaviors.
} 
\& Goldstein, 2003, Van Dalen \& Henkens, 2014, Moseley \& Stoker, 2015). Moreover, it has been acknowledged that 'default options in health information technology exert a powerful effect on user behavior...' (Malhotra et al., 2016, p. 891). ${ }^{3}$ For example, Malhotra et al. (2016) studies the redesign of an e-prescribing interface for medical providers where brand-name medications were substituted by generic equivalents during order entry (and allowing a one-click override to order the brand-name option) and found that the proportion of generic drugs prescribed increased from almost $40 \%$ to more than $95 \%$.

\section{Use of behavioral science on contact tracing app with exposure notifications during COVID-19}

Multiple models of contact tracing apps with exposure notifications have been employed during the pandemic, with differing degrees of user uptake. The MIT Technology Review COVID-19 Tracing Tracker project ${ }^{4}$ has documented a set of standard attributes pertaining to participation conditions, data management and underlying technology from apps in 46 countries (including only one in Latin America - Mexico), last updated in July 2020. The first multi-country large-scale study to measure attitudes toward contact tracing apps with exposure notifications to contain COVID-19 infections was performed in the UK, France, Germany, Italy, and the United States in March 2020 through a 10-minute online survey where respondents were presented with first with a hypothetical app that they could voluntarily install and then asked about the case in which the government requests mobile phone providers to automatically install the app on all phones, with the possibility to uninstall it (Altmann et al., 2020). ${ }^{5}$ The authors found broad support for a contact tracing app with exposure notification (70\% on average) - but while $74.8 \%$ of respondents stated that they would use the opt-in app, only $67.7 \%$ affirmed support for the opt-out model. They also found that those with lower trust in their national government are more hesitant to have the app on their phones. Among the main concerns related to exposure notification are possible government surveillance at the end of the pandemic and cybersecurity. ${ }^{6}$ In Australia, Bradshaw et al. (2020) conducted a $2 \times 2$ online experiment on a hypothetical application - measuring changes in frames (intention vs. obligation) and information storage (destroyed after 21 days and anonymous vs. being unsecured and kept forever). The authors found that the application framework does not make any difference, but specifying that the information is secure increases the desire to download the application and the probability of recommending it to a friend or family member. Trang et al. (2020) run a full-factorial 3

\footnotetext{
${ }^{3}$ Other examples of digital nudging in health (not using default options): Villani et al. (2013), Gonzalez and Dulin (2015), Carter et al. (2013), and Hammonds et al. (2015).

${ }^{4}$ Available at: https://www.technologyreview.com/tag/COVID-19-tracing-tracker/.

${ }^{5}$ This study was not an experiment - respondents were asked about both options, making its results not directly comparable to the experimental literature.

${ }^{6}$ Akinbi et al. (2020) highlight that the intrinsic mistrust of the government might make individuals think that a contact tracing app with exposure notifications could be the beginning of more pervasive government surveillance. Among other reasons are data storage and battery use concerns (Ayres et al., 2020).
} 
(benefit appeal: self vs. societal vs. self and societal) $\times 2$ (privacy design: low vs. high) $\times 2$ (convenience design: low vs. high) experiment in Germany during the rise of the COVID-19 pandemic by developing various designs for a fictitious app named COV-19 WATCH. They found that individuals characterized as 'critics' (at the 25 quantile of the propensity for accepting the app distribution) respond to societal-benefit appeals and privacy design in their installation decision. For 'undecided citizens' (at the 50 quantile of the propensity for accepting the app distribution), employing societal-benefit appeals and convenience in app usage is relevant. For 'advocates' (at the 75 quantile of the propensity for accepting the app distribution), none of the benefit appeals is superior. Finally, Ayres et al. (2020) conducted an experiment in the United States where in phase I, participants in the treatment groups were provided with information about a hypothetical app, and in phase II, they had to imagine that they had downloaded the app, and were exposed to different kinds of in-app notifications. They found that in-app notifications can be effective tools to promote pro-social behaviors and to induce people to take more precautions.

\section{Data}

The data for our analysis come from a phone survey conducted in ten countries in Latin America: Uruguay, Chile, Paraguay, Peru, Ecuador, Panama, Honduras, Costa Rica, El Salvador, and Mexico. In each country, 1000 individuals $^{7}$ over 18 years old randomly chosen answered a survey with an approximate duration of 18 minutes. The survey included questions regarding technology usage, trust, behavior, and COVID-19, as well as basic socio-demographic indicators. It contained a module on a hypothetical contact tracing app with exposure notifications from the national government that would not consume data and that would allow respondents to know if they have a COVID-19 symptom and what to do about it. There were two versions of the questionnaire, each with a different approach to the hypothetical app module, randomly distributed among participants within country. As such, for half of the respondents, the questions were about an app that had an opt-in regime (it could be download voluntarily) and, for the other half, the default was an opt-out regime where the app was going to be installed automatically on their phone, but they could voluntarily uninstall it. ${ }^{8}$ Examples of apps installed by default are Samsung/ Apple health, Google Maps, or Apple Music. While all those apps still require an agreement to terms and conditions to enable certain features (e.g., track heart rate or steps through Samsung/Apple health), they are similar to the idea that the questions in the survey were trying to capture. In fact, in some areas of the United States, the Apple operating system already included the contact tracing functionality, and users only had to turn it on as a setting to allow Public Health Authorities to notify you of possible exposure to COVID-19. ${ }^{9}$

\footnotetext{
${ }^{7}$ In Mexico, the sample was of 1200 individuals to make the survey representative at the state level.

${ }^{8}$ As a phone survey, the questions did not get into the specifics if the application would imply consent usage of data or if acknowledgement and agreement to terms and conditions was going to be required.

${ }^{9}$ For more details on the specifics of contact tracing apps with exposure notifications, see Nelson et al. (2021).
} 
The main outcome that we analyze in this article is self-reported intention to accept contact tracing apps based on whether the individual would voluntarily download it (opt-in) or voluntarily not remove it (opt-out) from their smartphone. See Supplementary Appendix Section B for details on data collection timeframes and survey design. For our analysis, we use the $73.8 \%$ of respondents who used a smartphone the week before the interview - as those are the only ones that answer the questions default options and acceptance of contact tracing apps with exposure notifications. In Supplementary Appendix Section C, we show the descriptive statistics for this sample and prove that there are no statistically significant differences in socio-demographic characteristics, smartphone activities, trust, and data protection concerns across treatment groups. Something to highlight is the overall low levels of trust in public institutions (only $16 \%$ of our sample reports having a lot of trust in the government), low levels of interpersonal trust (over $80 \%$ of respondents think you can never be careful enough in your interaction with others), weak data protection frameworks (77\% of Latin Americans claim that there are more risks than benefits of sharing personal data), and high levels of citizen concern regarding privacy in our sample (e.g., 58\% (69\%) of respondents claim not to know what private companies (the government) do with their personal data). Supplementary Appendix Table C.3 uses data from Latinobarometro to show how representative our sample is in terms of key variables.

\section{Empirical approach}

\section{Descriptive analysis}

As seen in Figure 1, 53\% of individuals self-report their intention to download the app with notifications in their phone (or already have the official one of their country) in the opt-in regime. In the opt-out, we can see that $72 \%$ of respondents selfreport they would not uninstall it (or that they already have it). In the other extreme, for not installing/uninstalling the app those figures are $18.4 \%$ and $11.1 \%$, respectively. In Supplementary Appendix Section D, we show the same analysis done for the basic app (with no exposure notification). By observing these differences in means, it seems that there would be more chances that the app is in the phone of Latin Americans if the option is opt-out rather than opt-in, as the differences that we describe here are significant at the $1 \%$ level. We can see that under the opt-in regime, intention to accept is the highest in Chile and Panama. Under the opt-out regime, the highest levels of self-reported intention to not remove the app are seen in El Salvador, Peru, and Uruguay. What we find differs from what was observed in Europe and the United States by Altmann et al. (2020). While in developed countries on average $70 \%$ of respondents report their intention to install or keep the app across regimes (under voluntary installation $74.8 \%$ of respondents claim to definitely install or probably install the app and under automatic download $67.7 \%$ of respondents claim to definitely keep or probably keep the app ${ }^{10}$ ), in Latin America, the magnitude of the effect of the opt-out option in increasing contact tracing app with exposure notification intention to accept is non-negligible. Two interrelated reasons may explain

\footnotetext{
${ }^{10}$ While Altmann et al. (2020) had five alternatives for self-reported intention to download or not uninstall the app, in our study there were three.
} 

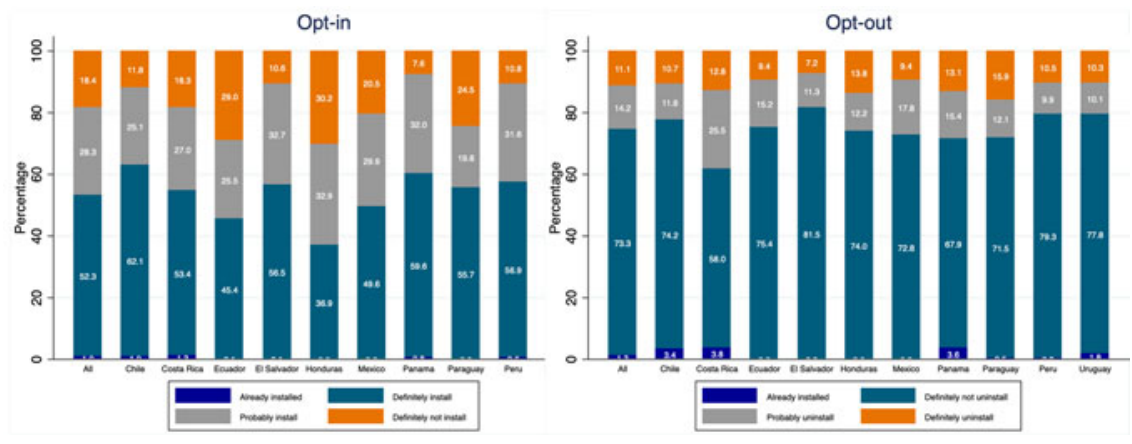

Figure 1. Acceptance rate of exposure notifications app, by country and regime. Note: for both regimes, survey allowed respondents to spontaneously respond that they already have downloaded the official app of their country.

this: different attitudes toward privacy and different levels of interpersonal trust. First, as shown by Prince and Wallsten (2020), Europeans generally place a higher value on privacy than Latin Americans. For example, they found that whereas Europeans and Americans demanded payment to receive personalized advertising via text message, participants from Argentina, Colombia, and Mexico were willing to pay for such advertising. This may lead Europeans and Americans to objecting more to an app that is automatically installed on their phone without prior consent. Second, European countries have higher levels of interpersonal trust than Latin American countries (Haerpfer et al., 2020). This potentially could lead to a belief that their fellow countrymen would actively make the pro-social decision of downloading a contact tracing app. This would make the opt-in version more effective on the aggregate, as the app's effectiveness depends on the scale of take-up. Europeans and Americans may also perceive the opt-in version as more palatable to their fellow countrymen for the privacy concerns mentioned above. Latin Americans, conversely, may perceive an app that is imposed from outside to be more effective than an opt-in one, due to their lower faith in others to actively make a pro-social decision. In Supplementary Appendix Section E, we show the determinants of acceptance per regime, and the self-reported reasons behind them.

The intention to accept question was later repeated but adding conditions to the contact tracing app with exposure notifications. First, we see the change in the intention to accept of the contact tracing apps with exposure notification separately under the two regimes if the person were a COVID-19 positive case, if the person had a family member who is COVID-19 positive, and if the app instead of being designed by the national/federal government were to be designed by the local government, an international tech company (like Apple or Google), a phone company or the WHO. Figure 2 shows the aggregate results. For each regime, thinking of having COVID-19 or having a family member that has tested positive for COVID-19 increases the self-reported intention to install (not uninstall) the app: around 65\% intention to accept in the opt-in case and $81 \%$ in the opt-out (both differences are significant, as shown in Supplementary Appendix Figure E.3). When compared to the national government, all the other potential designers (local governments, an 

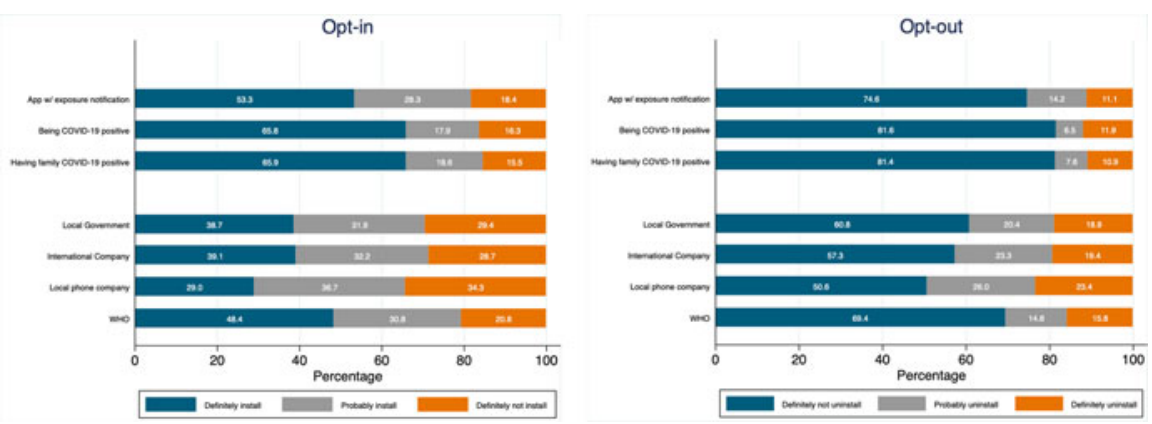

Figure 2. Acceptance rate of exposure notifications app under different conditions, by regime. Note: conditions include thinking about being COVID-19 positive, thinking about having a family member who is COVID-19 positive, and having the local government, an international company, a local phone company or the WHO design the app (rather than the national government).

international company, a local phone company, and the WHO) decrease intention to accept the app for each regime. For more information on the results of this survey and the implications for contact tracing app design, refer to Nelson et al. (2021).

\section{Model}

To estimate whether default options can increase the intention to accept a contact tracing app with exposure notifications, we run the following econometric model: $Y i=$ $\beta 0+\beta 1 T i+\gamma \mathbf{X} i+\epsilon i$, where $Y i$ is a dummy that equals to 1 if a respondent self-reports an intention to accept the contact tracing app with exposure notifications - claims to 'Definitely Install' or spontaneously states they already have the official app of the country in the opt-in regimen or 'Definitely not uninstall' in the opt-out regime (or spontaneously states that they already have the official app of the country), and 0 otherwise (note that to be conservative, a self-reported acceptance of the app is when the person claims for sure to either install it or not uninstall it). T1 is a dummy equal to 1 if subject $i$ was randomly assigned to the opt-out survey arm and 0 if she was assigned to the opt-in, and $\epsilon i$, is the error term. $\mathbf{X} i$ is a vector of covariates including gender, age, education, household composition, level of trust in the government, level of trust in other individuals, perception of data protection, and country that the respondent belongs to. As a second step, we also run the same series of analysis but conditioning the outcome to the alternatives in Figure 2. The model is run through OLS with robust standard errors and population weights.

\section{Results}

Figure 3 shows the results of the linear probability model for the effect of the opt-out regime versus the opt-in one. In every case, having a contact tracing app with exposure notification that is installed automatically significantly increases the intention to accept compared to an app that must be voluntarily installed - even after controlling for socioeconomic, level of trust, phone usage, and data control characteristics, as well 


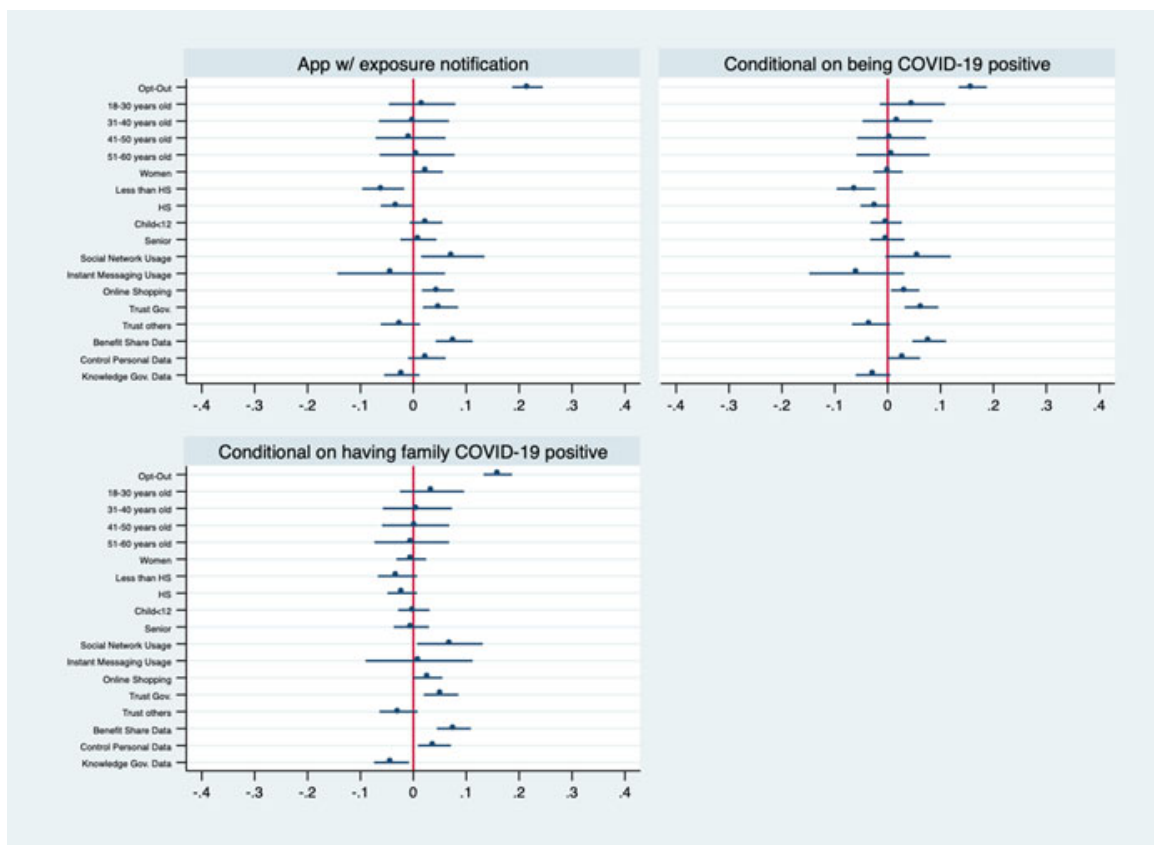

Figure 3. Main results. Note: in the case of opt-in, the dependent variable is an indicator variable taking the value 1 if a respondent answered 'Definitely Install' or spontaneously declared to have the official app of their country, and 0 for 'Probably Install' or 'Definitely not install'. In the case of opt-out, the dependent variable is an indicator variable taking the value 1 if a respondent answered 'Definitely not uninstall' (or spontaneously declared to have the official app of their country), and 0 for 'Definitely uninstall' or 'Probably uninstall'. We use a Linear Probability Model. Lines represent 95\% confidence intervals calculated with heteroskedasticity-robust standard errors. Population weights used. Marginal effects displayed (a coefficient of 0.1 implies a respondent who chose this option is 10 percentage points more likely to state they would use the phone relative to the base category). Base categories: 61 years old or more, more than high school; men; no children under 12 at home; no seniors at home; does not use social network or instant messaging; does not do online shopping; does not trust the government nor others; does not believe the benefits of sharing data outweight the costs; does not believe to have control over its personal data, or to know what the government does with personal data. Includes country FE. See Supplementary Appendix Table F.1 for point estimates, standard errors, significance levels and $N$.

as country fixed effects. In the case of the basic contact tracing app with exposure notifications, results show that the opt-out option significantly increases the intention to accept by 21.58 percentage points compared to the opt-in regime. Given that the self-reported intention to install the app is $52 \%$, the previous translates into an increase of around $40 \%$ in intention to accept. Such probability is 6 percentage points lower for those with less than high school education compared to respondents with more than high school; 3.1 percentage points lower for respondents with high school education compared to those with more than high school; 7.4 percentage points higher for those who regularly use instant messenger; 4.6 percentage points higher for those who do online shopping on their phones (compared to those who never do); 5 percentage points higher for those who trust the government; and 7.7 percentage points higher for those who believe the benefits of sharing data outweigh the 

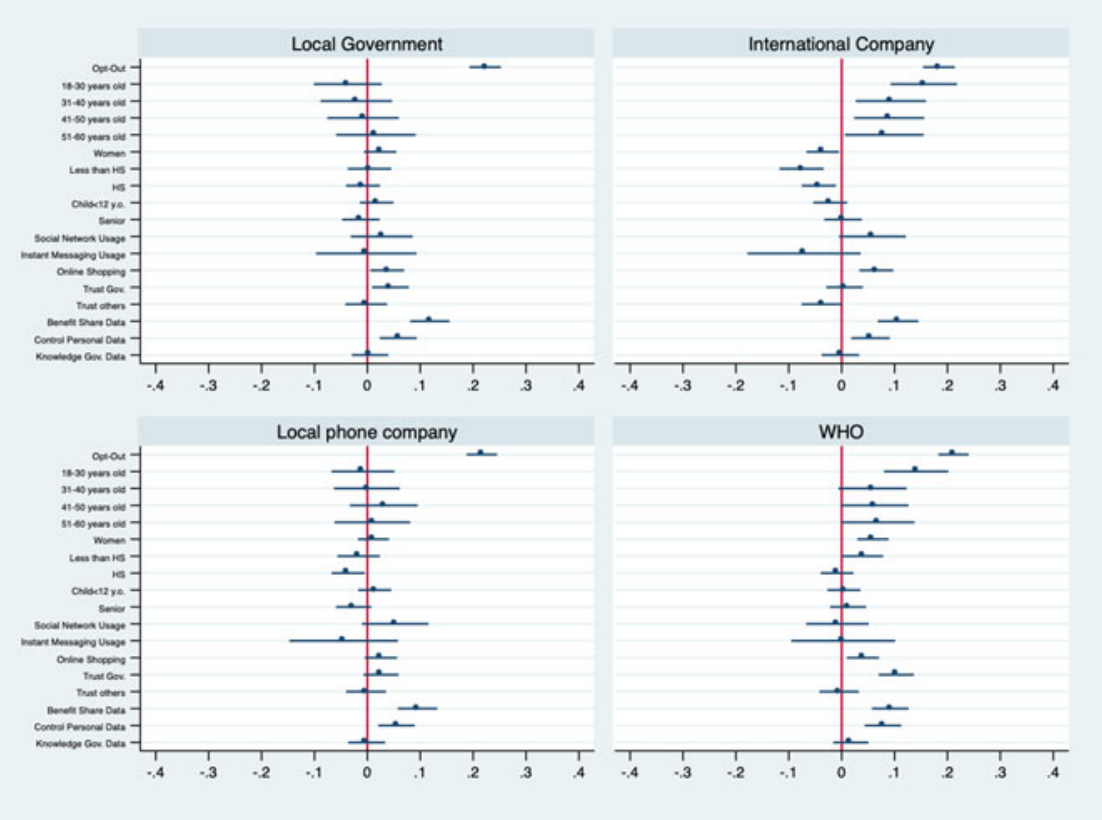

Figure 4. Main results, by implementing institutions (local government, international company, local phone company, WHO). Note: idem Figure 3.

risks. Conditional on thinking about being COVID-19 positive, the opt-out option significantly increases the intention to accept by 16.09 percentage points compared to the opt-in regime; the same figure is 15.98 percentage points when thinking about having a family member who is COVID-19 positive. The comparison of the regression coefficients between the unconditional contact tracing app option and each of the conditional ones shows that the difference in the point estimates is significant at the 1\% level. In Figure 4, we change the dependent variable to the app being designed by different institutions instead of the national government. When the local government designs it, results show that the opt-out option significantly increases the intention to accept in 22.28 percentage points compared to the opt-in regime. When the designer is an international company like Apple or Google, the difference is 18.30 percentage points (with younger cohorts, regularly using the phone for messaging or financial transactions, believing to have control over personal data and believing that sharing personal data has more benefits than costs increasing such probability, and being a woman and less educated decreasing it). On the other hand, when the designer is a local phone company, the figure is 21.64 percentage points, similar to the results for when the designer is the WHO (21.14 percentage points). The comparison of the regression coefficients between the app designed by the federal government and each of the other designer options shows that the only significant difference in point estimates is seen for an international company like Apple or Google. In general terms then, the opt-out regime increases the intention to accept contact tracing 
apps with exposure notifications. The difference in intention to accept between the regimes is reduced when respondents are prompted to think about being COVID-19 positive, have a family member who is COVID-19 positive or when the designer is an international company like Apple or Google rather than the national government. In the first two cases, the reason for the reduction of the difference between regimes is that the probability of acceptance under the opt-in option when the individuals feel at risk increases more than proportional than the probability of acceptance in the opt-out option under the same circumstance. However, the decrease in the difference between regimes in the last case is a product of the probability of acceptance under an implementing institution like Google or Apple (rather than the national government) decreasing under the opt-out option more than proportional than the decrease in probability of acceptance in the opt-in option under the same circumstances. See Supplementary Appendix Table F.1 for the estimates of all different models. ${ }^{11}$

In Supplementary Appendix Section F.3, we performed a series of heterogeneous effects. While, in every case, the intention to accept the app increases with an opt-out regime compared to an opt-in one, we do not find statistically significant differences by region within Latin America or by COVID-19 incidence. Moreover, while we found some indication that default options could be more effective for those who do not trust the government, we do not find any differences by the degree of control over personal data. Given that the previous issues of trust and data sharing concerns are self-reported perceptions, we finally show results by phone usage to make financial transactions (do online shopping or payments). The assumption is that reporting using phones regularly to make transaction is a better proxy for actual behavior related to data privacy concerns. We found that the probability of acceptance of the app increases by 24.9 percentage points in the case of an opt-out regime compared to an opt-in for respondents who claim to never have used their phones in the previous week to make financial transactions. For those who did, such figure is reduced by 9.12 percentage points. We can thus extrapolate that default options are more effective for those who, probably, are not comfortable with having bank or credit card information to make payments through their phone - in other words, those who by their actions show not feel comfortable sharing personal data compared to those who do.

Finally, in Supplementary Appendix Section F.4, and as a robustness test for our previous results, we also run the same analysis but changing the outcome, exploiting three questions related to the handling of the pandemic that were asked after the contact tracing app with exposure notification module. We do not find significant differences by treatment arm.

\section{Conclusion}

Testing, contact tracing and quarantine/isolation are the key strategies to contain the spread of an infectious disease for which vaccination is not yet (widely) available.

\footnotetext{
${ }^{11}$ Supplementary Appendix Section F.2 shows the analysis excluding those who already downloaded the official apps of their country and find similar results.
} 
Given the rate of transmission of COVID-19, relying only on manual contact tracing might be challenging to control the spread of the virus. Contact tracing apps with exposure notifications in theory, and under the right protocols, have the potential benefits of being limited to physical proximity only, of not relying on individual recall, of being anonymous by design, of allowing an immediate alert and not requiring human inputs for contact identification. Such features can be important in Latin America, where, as shown in this article, there are low levels of trust in public institutions, high levels of citizen concern regarding privacy, generally weak data protection frameworks, and low levels of interpersonal trust.

In this article, we found that there is an overall high reported intention to accept contact tracing apps with exposure notifications - around $64 \%$ on average across opt-in (voluntary installation) and opt-out (automatic installation with an uninstallation option) regimes. This figure is slightly lower to what was found in Europe and the United States, which was $70 \%$ on average. Acceptance increases if the person thinks about being COVID-19 positive or having a family member who is infected. Moreover, most participants stated that they trusted an app designed by national governments more than one designed by local governments, local phone companies, international companies, or the WHO.

The main finding of this study is that framing matters: the opt-out option significantly increases the intention to accept a contact tracing app with exposure notification by 21.58 percentage points compared to the opt-in regime (which translates into an average increase of around 40\%), even after controlling for individual characteristics. This result is very much aligned with findings from many areas in behavioral economics studies, such as the use of green energy, organ donation and savings (Susntein, 2020). It is also aligned with the findings of Malhotra et al. (2016) regarding how combining default options with technology in the health sector can leverage the powerful effects that default options already have. However, our results differ from those found in developed countries, potentially due to lower concerns regarding privacy and lower levels of interpersonal trust in Latin America. Moreover, in our sample, the difference between regimes is reduced when respondents have to think about being COVID-19 positive, have a family member who is COVID-19 positive or when the designer is an international company like Apple or Google rather than the national government. It is also reduced when respondents claim to have used their phones in the previous week to make financial transactions compared to those who do not, and for those who trust the government compared to those who do not. In terms of individual characteristics, we also find that trust is important for acceptance, as well as using a cellphone for financial transactions and believing that the benefits of sharing personal data outweigh the risks.

Our study has some limitations that we tried to address through the article. First, our data was collected on a sample of Latin American countries, so results might not apply to other regions. We do find, however, that for every country in the sample an opt-out regime significantly increases self-reported acceptance compared to an opt-in option (though magnitudes vary). Therefore, our evidence-based policy recommendations can be applied through the continent and might put us one step closer to ending the pandemic. Second, we cannot observe actual behavior and therefore whether that translates into observable actions is beyond the scope of this article. 
However, the severity and urgency of the COVID-19 pandemic means that several studies, like ours, have relied on self-reported behaviors and perceptions to provide policy analysis recommendations. Moreover, previous research projects have found a good correlation between self-reported and actual behavior (see, e.g., Chandon et al., 2005; Junco, 2013; Hainmueller et al., 2015; Sticca et al., 2017; Waterfield et al., 2020). Third, research has found that uninstall rates for apps is around $28 \%$ after 30 days of app installation (Freer, 2018), so perceptions and attitudes toward the app months after its introduction are not being captured by our survey. Finally, in our article, we measured support for the 'general concept' of a contact tracing app, so not having all the details might have influenced responses. However, the fact that the main reasons reported by individuals regarding their unwillingness to accept the app seem to be the same across regimes suggests that our concept is acceptable for this study. ${ }^{12}$

In terms of what our findings imply for policymakers, several things are worth highlighting. First, contact tracing apps with exposure notifications are not a silver bullet, are still being tested, and need to be part of a broader strategy. For example, apps must be supported by massive testing so that notifications do actually take place. The risk of having a contact tracing app with exposure notification in people's smartphones without these underlying conditions is creating a feeling of false security, as positive cases will go undetected, and alerts will never be sent - which might induce them to act irresponsibly (also highlighted by Ayres et al. (2020)). Moreover, there is still the need for those who get a positive notification to quarantine.

Second, there are practical implications to consider. For instance, there needs to be a Governmental body in charge of decisions such as: how will positive cases be notified?, what is considered significant contact? (how many minutes? at what distance?) how many people have smartphones? what models are more common? (old models may not have the necessary requirements). Moreover, sunset clauses (the app would be automatically deleted from smartphones once the pandemic is over) as well as a data destruction mechanism are also issues that have been considered when thinking about default options (Dahmm, 2020; Miller, 2020). Policymakers along mobile phone companies would also have to work together to determine the best way to preinstall these apps, with the before mentioned technical considerations.

Third, there is a possible paradoxical risk of success in acceptance of contact tracing app with exposure notifications: if Latin American countries are successful in prompting high uptake, then due to the weak institutional capacity regarding data protection (Muente \& Serale, 2020), there is greater exposure to misuse of personal data perhaps even undermining the utility of such apps before the pandemic is over. This means that, even if high adoption of contact tracing apps with exposure notifications is necessary for fighting the pandemic, it carries a new (additional) need for strengthening data protections in the region. That is one of the reasons why models like the one promoted by Google and Apple are preferred to centralized options that need users to register with the authority providing the app.

\footnotetext{
${ }^{12}$ Altmann et al. (2020) found that details given about implementation (like if the app used Bluetooth or GPS) had little impact on support.
} 
Therefore, finally, we must find a balance. There is a clear tradeoff between facilitating uptake of these apps while still maintaining that the population is well informed and providing explicit consent to use these applications. Defaults may increase acceptance significantly - as was shown in this article through self-reported willingness to accept - but may potentially reduce perceptions around trust and transparency. If people do not trust the technology or the entity that deploys it, they may look for workarounds (i.e., leave their devices at home) or not report for testing, which would jeopardize the effectiveness of the efforts to stop the spread of the disease. We show in our article that issues of trust like not wanting the government to have access to their location, not believing in the promises of anonymity or thinking the government could have control over people, as well as technical reasons like not having enough space on their phone or not knowing how to uninstall the app are of concern among respondents that were asked about the hypothetical opt-out app (even though, as we noted, those concerns could have been influenced by the fact that we asked about the 'general concept' of a contact tracing app with little details). The previous, plus legal requirements for explicit consent, need to be considered [see companion paper Nelson et al. (2021)], and therefore, it may be difficult to implement a pure opt-out option. However, we cannot ignore the fact that explicit consent may reduce uptake. Policymakers should consider that many aspects of a contact tracing app with exposure notification can benefit from the use of defaults, all while maintaining privacy by design, explicit consent for key functions following legal guidelines, and clear communication to citizens. Moreover, it is important for policymakers to think of ways of extending the use of these apps to deal with other aspects of the pandemic, like increasing vaccination efforts [e.g., having automatic vaccination appointments, similar to the experiment performed by Chapman et al. (2010) with the influenza vaccine]. These aspects are critical to ensure that users trust the technology and, overall, the health system to effectively influence behaviors for current and future pandemics in Latin America.

Supplementary material. To view supplementary material for this article, please visit https://doi.org/10. 1017/bpp.2021.38.

Acknowledgements. This article could not have been possible without the research assistance of Juan Manuel Monroy Barragan and Neili Bermudez. We would also like to thank the participants of LACEA BRAIN and the valuable feedback we received from Jennifer Nelson, Arturo Muente, and Florencia Serale from the Inter-American Development Bank, and from two anonymous referees.

Funding statement. The Inter-American Development Bank (IDB) provided funding for data collection. The funder also provided support in the form of salaries for authors Florencia Lopez Boo, Luis Tejerina, and Benjamin Roseth, but did not have any additional role in the study design, data collection and analysis, decision to publish, or preparation of the manuscript.

Competing interest. The authors declare that they have no conflict of interest.

\section{References}

Abeler, J., M. Bäcker, U. Buermeyer and H. Zillessen (2020), 'COVID-19 contact tracing and data protection can go together', JMIR mHealth and uHealth, 8(4): e19359.

Abueg, M., R. Hinch, N. Wu, L. Liu, W. J. Probert, A. Wu, P. Eastham, Y. Shafi, M. Rosencrantz, M. Dikovsky and Z. Cheng (2020), Modeling the combined effect of digital exposure notification and non-pharmaceutical interventions on the COVID-19 epidemic in Washington state. medRxiv. 
Akinbi, A., M. Forshaw and V. Blinkhorn (2020), Contact tracing apps for COVID-19 pandemic: Challenges and potential. Retrieved from: https://osfio/6xbcs.

Altmann, S., L. Milsom, H. Zillessen, R. Blasone, F. Gerdon, R. Bach, F. Kreuter, D. Nosenzo, S. Toussaert and J. Abeler (2020), 'Acceptability of app-based contact tracing for COVID-19: Cross-country survey study', JMIR mHealth and uHealth, 8(8): e19857.

Anglemyer, A., T. H. Moore, L. Parker, T. Chambers, A. Grady, K. Chiu, M. Parry, M. Wilczynska, E. Flemyng and L. Bero (2020), 'Digital contact tracing technologies in epidemics: A rapid review', Cochrane Database of Systematic Reviews, 8(2020): 1-42. Article CD013699.

Ayres, I., A. Romano and C. Sotis (2020), How to make COVID-19 contact tracing apps work: Insights from behavioral economics. Available at SSRN 3689805.

Bay, J., J. Kek, A. Tan, C. S. Hau, L. Yongquan, J. Tan and T. A. Quy (2020), BlueTrace: A privacy-preserving protocol for community-driven contact tracing across borders. Government Technology Agency-Singapore, Tech. Rep.

Bernheim, B. D., A. Fradkin and I. Popov (2015), 'The welfare economics of default options in 401 (k) plans', American Economic Review, 105(9): 2798-2837.

Bradshaw, E. L., R. Ryan, M. Noetel, A. K. Saeri, P. Slattery, E. Grundy and R. A. Calvo (2020), Information safety assurances affect intentions to use COVID-19 contact tracing applications, regardless of autonomy-supportive or controlling message framing. Retrieved from: https://osf.io/5wap8.

Carter, M. C., V. J. Burley, C. Nykjaer and J. E. Cade (2013), 'Adherence to a smartphone application for weight loss compared to website and paper diary: Pilot randomized controlled trial', Journal of Medical Internet Research, 15(4): e32.

Chandon, P., V. G. Morwitz and W. J. Reinartz (2005), 'Do intentions really predict behavior? Self-generated validity effects in survey research', Journal of Marketing, 69(2): 1-14.

Chapman, G. B., M. Li, H. Colby and H. Yoon (2010), 'Opting in vs opting out of influenza vaccination', Journal of the American Medical Association, 304(1): 43-44.

Choi, J. J., D. Laibson and B. C. Madrian (2004), 'Plan design and 401 (k) savings outcomes', National Tax Journal, 57(2): 275-298.

Dahmm, H. (2020), Data sharing in a post-pandemic world: how to safely wind down surveillance measures. SDG Knowledge HUB. Retrieved from: https://sdg.iisd.org/commentary/guest-articles/datasharing-in-a-post-pandemic-world-how-to-safely-wind-down-surveillance-measures/.

DP-3T Project (2020), Privacy and security risk evaluation of digital proximity tracing systems. Retrieved from: https://github.com/DP-3T/documents.

Everett, J. A., C. Colombatto, V. Chituc, W. J. Brady and M. Crockett (2020), The effectiveness of moral messages on public health behavioral intentions during the COVID-19 pandemic. PsyArXiv Preprints. Retrieved from: https://doi.org/10.31234/osf.io/9yqs8.

Ferretti, L., C. Wymant, M. Kendall, L. Zhao, A. Nurtay, L. Abeler-Dörner, M. Parker, D. Bonsall and C. Fraser (2020), 'Quantifying COVID-19 transmission suggests epidemic control with digital contact tracing', Science, 368(6491): 1-7.

Feuer, W. (2020), May 22. South America is a 'new epicenter' of the coronavirus pandemic, WHO says. CNBC. Retrieved from: https://www.cnbc.com/2020/05/22/south-america-is-a-new-epicenter-of-thecoronavirus-pandemic-who-says.html.

Freer, A. (2018), Mobile app uninstall rate after 30 days is $28 \%$ according to AppsFlyer. Business of Apps. Retrieved from: https://www.businessofapps.com/news/mobile-app-uninstall-rate-after-30-days-is-28according-to-appsflyer/.

Frimpong, J. A. and S. Helleringer (2020), Financial incentives for downloading COVID-19 digital contact tracing apps. Retrieved from: https://osf.io/preprints/socarxiv/9vp7x/.

Glöckner, A., A. R. Dorrough, T. Wingen and S. Dohle (2020), The perception of infection risks during the early and later outbreak of COVID-19 in Germany: Consequences and recommendations. PsyArXiv Preprints. Retrieved from: https://psyarxiv.com/wdbgc/.

Gonzalez, V. M. and P. L. Dulin (2015), 'Comparison of a smartphone app for alcohol use disorders with an Internet-based intervention plus bibliotherapy: A pilot study', Journal of Consulting and Clinical Psychology, 83(2): 335.

Haerpfer, C., R. Inglehart, A. Moreno, C. Welzel, K. Kizilova, J. Diez-Medrano, M. Lagos, P. Norris, E. Ponarin, B. Puranen, et al. (eds) (2020), World Values Survey: Round Seven - Country-Pooled 
Datafile. Madrid, Spain \& Vienna, Austria: JD Systems Institute \& WVSA Secretariat. doi:10.14281/ 18241.13.

Hainmueller, J., D. Hangartner and T. Yamamoto (2015), 'Validating vignette and conjoint survey experiments against real-world behavior', Proceedings of the National Academy of Sciences, 112(8): 2395-2400.

Halpern, S. D. (2018), 'Using default options and other nudges to improve critical care', Critical Care Medicine, 46(3): 460.

Halpern, S. D., G. Loewenstein, K. G. Volpp, E. Cooney, K. Vranas, C. M. Quill, M. S. McKenzie, M. O. Harhay, N. B. Gabler, T. Silva and R. Arnold (2013), 'Default options in advance directives influence how patients set goals for end-of-life care', Health Affairs, 32(2): 408-417.

Hammonds, T., K. Rickert, C. Goldstein, E. Gathright, S. Gilmore, B. Derflinger, B. Bennett, A. Sterns, B. L. Drew and J. W. Hughes (2015), 'Adherence to antidepressant medications: A randomized controlled trial of medication reminding in college students', Journal of American College Health, 63(3): 204-208. Johnson, E. J. and D. Goldstein (2003), 'Do defaults save lives?' Science, 302(5649): 1338-1339.

Johnson, E. J., J. Hershey, J. Meszaros and H. Kunreuther (1993), 'Framing, probability distortions, and insurance decisions', Journal of Risk and Uncertainty, 7(1): 35-51.

Johnson, E. J., S. Bellman and G. L. Lohse (2003), 'Cognitive lock-in and the power law of practice', Journal of Marketing, 67(2): 62-75.

Junco, R. (2013), 'Comparing actual and self-reported measures of Facebook use', Computers in Human Behavior, 29(3): 626-631.

Just, D. and J. Price (2013), 'Default options, incentives and food choices: Evidence from elementary-school children', Public Health Nutrition, 16(12): 2281-2288.

Kaiser, M., M. Bernauer, C. R. Sunstein and L. A. Reisch (2020), 'The power of green defaults: The impact of regional variation of opt-out tariffs on green energy demand in Germany', Ecological Economics, 174 (106685): 1-12.

Lunn, P. D., S. Timmons, H. Julienne, C. A. Belton, M. Barjaková, C. Lavin and F. P. McGowan (2021), 'Using decision aids to support self-isolation during the COVID-19 pandemic', Psychology \& Health, 36(2): 195-213.

Malhotra, S., A. D. Cheriff, J. T. Gossey, C. L. Cole, R. Kaushal and J. S. Ancker (2016), 'Effects of an e-Prescribing interface redesign on rates of generic drug prescribing: Exploiting default options', Journal of the American Medical Informatics Association, 23(5): 891-898.

Miller, K. (2020), Is an Opt-Out Contact-Tracing App the Best Way to End the Pandemic? Human Centered Artificial Intelligence (HAI), Stanford University. Retrieved from: https://hai.stanford.edu/ news/opt-out-contact-tracing-app-best-way-end-pandemic.

Moseley, A. and G. Stoker (2015), 'Putting public policy defaults to the test: The case of organ donor registration', International Public Management Journal, 18(2): 246-264.

Muente, A. and F. Serale (2020), Uso de datos personales durante la pandemia. Washington, DC: Mimeo.

Nelson, J., L. Goyeneche, N. Bermudez, C. Boruchowicz, F. Lopez Boo, J. M. Monroy, B. Roseth and L. Tejerina (2021), Pandemics, privacy, and technology adoption: Perceptions of the use of digital tools during COVID19 from 10 Latin American Countries. Mimeo.

Park, C. W., S. Y. Jun and D. J. MacInnis (2000), 'Choosing what I want versus rejecting what I do not want: An application of decision framing to product option choice decisions', Journal of Marketing Research, 37(2): 187-202.

Prince, J. and S. Wallsten (2020), How Much is Privacy Worth Around the World and Across Platforms?. Available at SSRN. Retrieved from: https://techpolicyinstitute.org/wp-content/uploads/2020/01/ Prince_Wallsten_How-Much-is-Privacy-Worth-Around-the-World-and-Across-Platforms.pdf.

Reuters (2021), Reuters COVID-19 Tracker, Latin America and the Caribbean. Available at: https://graphics.reuters.com/world-coronavirus-tracker-and-maps/regions/latin-america-and-the-caribbean/.

Roseth, B. and M. Porrúa (2021), Trust and digital transformation. Mimeo.

Roseth, B., A. Reyes, P. Farias, M. Porrúa, H. Villalba, S. Acevedo and P. Fillotrani (2018), Wait No More: Citizens, Red Tape, and Digital Government. Washington, DC: Inter-American Development Bank.

Servick, K. (2020), 'Can phone apps slow the spread of the coronavirus?', Science, 368(6497): 1296-1297.

Sticca, F., T. Goetz, M. Bieg, N. C. Hall, F. Eberle and L. Haag (2017), 'Examining the accuracy of students' self-reported academic grades from a correlational and a discrepancy perspective: Evidence from a longitudinal study', PLoS One, 12(11): e0187367. 
Sunstein, C. R. and L. A. Reisch (2014), 'Automatically green: Behavioral economics and environmental protection', Harvard Environmental Law Review, 38(1): 127-158.

Susntein, C. R. (2020), Behavioral Science and Public Policy. Cambridge: Cambridge Elements Public Economics, Cambridge University Press.

Trang, S., M. Trenz, W. H. Weiger, M. Tarafdar and C. M. Cheung (2020), 'One app to trace them all? Examining app specifications for mass acceptance of contact-tracing apps', European Journal of Information Systems, 29(4): 415-428.

Troncoso, C., M. Payer, J. P. Hubaux, M. Salathé, J. Larus, E. Bugnion, W. Lueks, T. Stadler, A. Pyrgelis, D. Antonioli and L. Barman (2020), Decentralized privacy-preserving proximity tracing. https://arxiv. org/abs/2005.12273.

Van Dalen, H. P. and K. Henkens (2014), 'Comparing the effects of defaults in organ donation systems', Social Science \& Medicine, 106(4): 137-142.

Villani, D., A. Grassi, C. Cognetta, D. Toniolo, P. Cipresso and G. Riva (2013), 'Self-help stress management training through mobile phones: An experience with oncology nurses', Psychological Services, 10(3): 315.

Wang, C. J. (2021), 'Contact-tracing app curbs the spread of COVID', Nature, 594(7863): 336-337.

Waterfield, G., S. Kaplan and D. Zilberman (2020), 'Willingness to pay versus willingness to vote: Consumer and voter avoidance of genetically modified foods', American Journal of Agricultural Economics, 102(2): 505-524.

Wise, T., T. D. Zbozinek, G. Michelini, C. C. Hagan and D. Mobbs (2020), 'Changes in risk perception and self-reported protective behaviour during the first week of the COVID-19 pandemic in the United States', Royal Society Open Science, 7(9): 200742.

Wymant, C., L. Ferretti, D. Tsallis, M. Charalambides, L. Abeler-Dörner, D. Bonsall and C. Fraser (2021), 'The epidemiological impact of the NHS COVID-19 App', Nature, 594(7863): 408-412.

Cite this article: Boruchowicz C, Lopez Boo F, Roseth B, Tejerina L (2021). Default options: a powerful behavioral tool to increase COVID-19 contact tracing app acceptance in Latin America? Behavioural Public Policy 1-17. https://doi.org/10.1017/bpp.2021.38 